\title{
Field enhancement on nano-structures inside dielectrics
}

\author{
Kazem Ardaneh, Remo Giust, Francois Courvoisier \\ FEMTO-ST Institute, Univ. Bourgogne Franche-Comté, UMR CNRS 6174 \\ $15 B$ avenue des Montboucons, 25030 Besançon, France
}

The interaction between ultrafast laser pulses and the condensed matter has opened new frontiers in the physics and technology of light-matter interactions and laser micro-machining. The focusing of intense ultrafast laser pulses inside dielectric materials has led to the formation of nano-voids [1] and periodic nano-gratings. In the former case, the formation of dense plasma allows the excitation of electron plasma waves along the density gradient that can lead to the resonance absorption process. In the latter case, however, diffraction by the grating matches the incident laser energy into surface electromagnetic waves, known as surface plasmons, which propagate along the surface. In both cases, high energy absorption can be obtained. The electron plasma waves and surface plasmons are, therefore, unique properties in nano-structures for manipulating and confining the lasers.

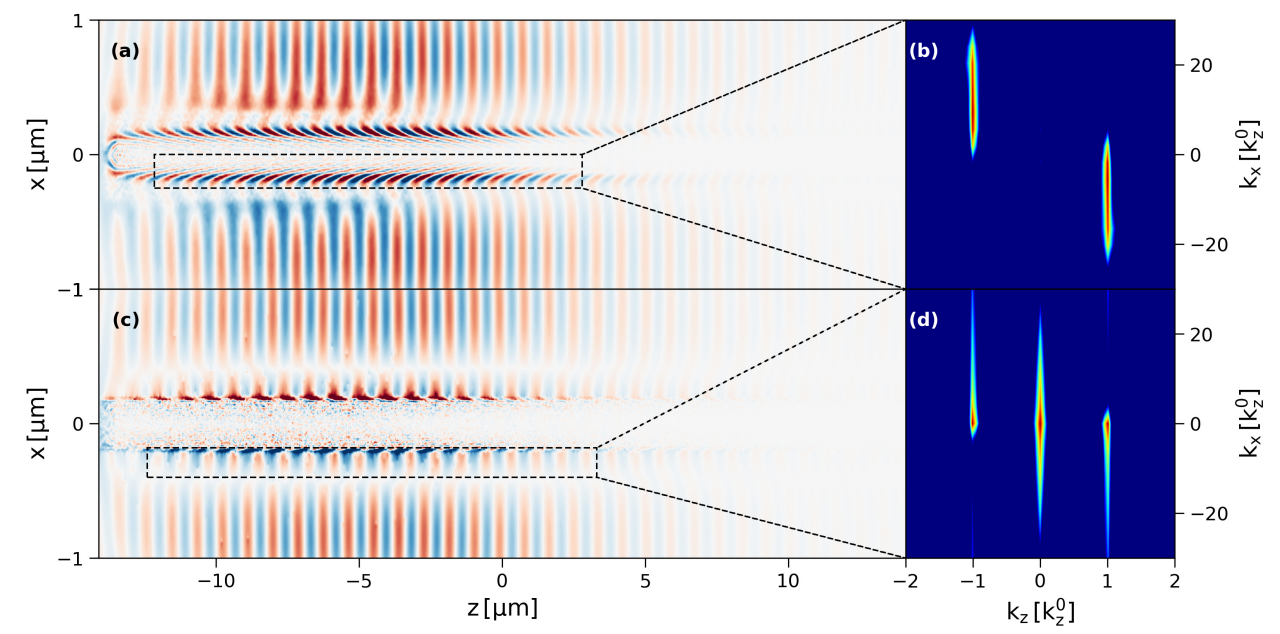

Figure 1: The results of PIC simulation for inhomogeneous plasma (top row), and homogeneous plasma (bottom row). The right column shows the 2D Fourier transformations performed on the dashed boxes.

In this theoretical work, we aim to explain the high energy absorption observed experimentally in focusing of a single $100 \mathrm{fs}$ Bessel pulse at central wavelength $800 \mathrm{~nm}$ inside the bulk of transparent dielectrics at intensities of $10^{14} \mathrm{Wcm}^{-2}$. We have performed Particle-In-Cell (PIC) simulations using EPOCH code [2]. Two density structures are considered for the over-critical plasma: inhomogeneous, and homogenous. In the case of the inhomogeneous, we have seen the propagation of the electron plasma waves along the density gradient (Figure 1a). The wave in this case has an oblique structure $\left(k_{\mathrm{x}} \neq 0, k_{\mathrm{z}} \neq 0\right)$ consisting of the laser wave and an electrostatic plasma wave (Figure $1 \mathrm{~b})$. One can see the formation of electromagnetic surface plasmon $\left(k_{\mathrm{x}} \approx 0\right)$ in the case of the homogenous plasma distribution (Figure 1c) in which the wave has an electromagnetic nature (Figure 1d). In both cases, absorption of 40 percent of the laser energy is measured, and excellent agreement is obtained with our experimental results. The plasma distribution however largely impacts on the heating and acceleration of the free electrons. The specific structure of the electron plasma waves in the inhomogeneous plasma is prone to accelerate electrons that can escape the plasma. In contrast, the heating of the homogeneous plasma is much more confined, which allows reaching higher temperatures of the plasma

In conclusion, PIC simulations could shed light on the microphysics of nano-plasma heating by an ultrafast Bessel pulse. We anticipate our results will impact the design of novel strategies for laser materials processing.

This research has received funding from H2020 European Research Council (ERC) under grant agreement 682032-PULSAR. This work was granted access to HPC resources PRACE (PULSARPIC PRA19_4980), TGCC (A0070511001), and Mésocentre de Calcul de Franche-Comté.

\section{References}

[1] M. K. Bhuyan, F. Courvoisier, P. A. Lacourt, et al, "High aspect ratio nanochannel machining using single shot femtosecond Bessel beams," Appl. Phys. Lett 97, 081102 (2010).

[2] T. D. Arber, K. Bennett, C. S. Brady, et al, "Contemporary particle-in-cell approach to laser-plasma modelling," Plasma Phys. Control. Fusion 57, 113001 (2015). 